\title{
Research of General Software Maintenance Management
}

\author{
Jing Li \\ Xi'an Peihua University, Xi’an, Shaanxi, 710125
}

Keywords: general software; maintenance management; data file

\begin{abstract}
With the rapid development of technology, computers have become closely related to our lives. For the use of computers, in addition to understanding the correct use, it is necessary to understand the daily management and maintenance of the computer. The hardware and software of a computer are two parts that are indispensable for a computer. Computer software refers to data files and programs in computer programs. Software is an important basis for the normal operation of computers. Therefore, when using computer software, it is essential to conduct in-depth research on the daily management and maintenance of computer software.
\end{abstract}

\section{Introduction}

For those who use computers, the maintenance and management of computer software is very important. In the use of various types of computers, if you do not pay attention to the use and maintenance of the computer, some of the performance of the computer will be difficult to fully play, resulting in some work can not be completed normally and will also affect the life of the computer. Therefore, it is necessary to strengthen the daily management and maintenance of the computer. We should ensure the computer performance is fully realized through real-time management and maintenance, so as to extend the service life of the computer.

\section{Common Problems of Computer Software}

The security maintenance system of the Internet itself is very low. It is difficult for the current Internet threat to have a substantial effect. In particular, it is still a very open system. Due to the use of the Internet, many problems and vulnerabilities have been discovered. The security of the Internet is not considered, resulting in the expansion of Internet information, the intrusion of bad information and viruses, resulting in slower computer software running. Software mainly refers to system software and application software. In the process of using the system software, if the user does not clean and maintain the system in time, the system will automatically generate a lot of junk files. Junk files not only take up system space, but also have a strict hindrance to the daily operation of the system. In the end, the system may run slower or even fail to operate and work normally. The means of computer software programming today are diverse. These technologies are generally mature. However, some computer software programming techniques in China have some shortcomings. Some of them exist in the software system. The problem is that it is easy to cause other problems during the use process, which affects the normal use of the network by Internet users. Computer software and the Internet are a platform for users to experience and communicate. Internet users can maintain and manage them in the process of using computer software. This is an important measure to improve their performance, but it may also bring computer software. Come with some unexpected effects and damage. Some users have different requirements for computer software, so there may be some difficulties and problems when using them. Software maintenance also requires a formal maintenance team to complete, requiring a clear division of labor and responsibility, so as to avoid unnecessary disputes. Before the computer software is maintained and managed, the user can create a request form to describe the errors in detail and comprehensively. According to the form provided by the user, the type of the problem, the time when the problem occurs, the target to be achieved, The environment in which the system operates, etc., and use this to find an effective solution to the problem. 


\section{Characteristics of Computer Software Maintenance and Management}

Give the computer a good, safe and healthy operating environment. It classifies the computer software according to different operation modes or functions of the computer software, and classifies the related computer software into a disk, which is convenient for management and modification when the computer runs. At the same time, there should be enough free space in the disk for storing the computer software system, so that the computer software system can use a separate area separately, effectively improving the efficiency of the computer software system, and also making the user Management is faster and easier!

Use the correct method to operate the computer software and manage it reasonably. First, do not modify or delete the system files of the computer at will. Computer system files are the guarantee that the computer software system can run normally. If you arbitrarily delete it, it may cause the computer software system to crash. Computer software itself always has unforeseen defects that cannot be avoided. These defects can only be gradually revealed under the test of time. Therefore, it is necessary to update the computer software in time to ensure safety and then use it. The viable method of these unforeseen software defects.

For computer viruses, users should frequently upgrade security patches. According to the data, $80 \%$ of network viruses are spread by system security vulnerabilities. Users regularly download the latest security patches to prevent problems before they happen. When performing computer operations, the user promptly shuts down or deletes unnecessary services in the system. By default, many systems will install some auxiliary services. These services are not very useful for ordinary users, but can be attacked. Computer users provide convenience. If these services are deleted in time, the possibility of being attacked can be effectively reduced, and normal operation will not be affected. In operation, remember to use some complicated passwords as much as possible to improve the security factor of your computer!

In order to make the network application itself have a certain degree of robustness and not be arbitrarily hacked, the user should first confirm whether the user identity of the accessed program is legal, and the user name and password should be verified. Secondly, the user's use should be considered. Permissions, allowing users who are verified to be legitimate to perform computer software operations within the scope of legal control; again, every page accessed by the user should be combined with previous passwords and permissions. Web applications are made in a remote environment, so be sure to pay attention to the user's secure submission of data.

\section{Ways to Maintain and Manage Computer Software}

Reasonable distribution should be based on the type and function of different software, and the software with strong correlation should be placed on the same disk to facilitate the management and modification of the software in the future. It is best to set up a separate storage area for the computer software, and to ensure that there is enough space in this area, on the one hand, it is beneficial to improve the operating efficiency of the software, on the one hand, it is beneficial to the user to maintain and manage the computer software system.

First, users should avoid arbitrarily modifying and deleting system files while using computer software. The system file of the software is the basic guarantee for the normal operation of the computer system. Once a tiny file is modified or deleted, it may cause the entire computer system to crash. Secondly, it is necessary to use the editor to manually modify the registry of the computer to achieve the effect of hiding some functional units and drive icons in the control panel, thereby effectively preventing some system files in the computer from being illegally operated. In addition, installing and using some secure computer system upgrade programs on the computer also facilitates the safe operation of the computer software system. Finally, for some security risks that exist in the calculator software system, regular or irregular virus detection should be carried out, and timely update should be carried out. After ensuring the security risks are effectively eliminated, the computer software will be started.

In the process of using the calculator, a strong virus defense mechanism should be established. 
By strengthening the powerful setting of the computer system related authority and the regular and irregular security maintenance work of the computer software system, the entire computer software system can be established. A defense system against malicious attacks such as viruses.

In addition to the compatibility of the computer system itself, the security maintenance of the computer software plays a vital role in the normal operation of the computer. If the security maintenance is not in place, it will affect the normal operation of the computer and the normal work of people. And life, so it is very important to strengthen software security maintenance. In the aspect of strong software security maintenance, it is crucial for the defense of computer viruses. If there is no special system or setting to defend against software viruses, it is easy for hackers and viruses to attack computer systems, resulting in computer systems. Oh, then you have to lose more. Strengthening the security maintenance of computer software involves many aspects and approaches. (1) Effectively defend against possible viruses and security problems, use the advantages and strengths of computer systems, and conduct regular analysis on the system itself. Once the analysis results are abnormal, it means that the computer system may be short in the future. When there is a security problem in time, it is necessary to use the computer's own defense system or a special defense system to protect the computer system, so as to effectively avoid computer security problems, stop the security problem from the source; (2) users should strengthen Safety awareness, when using computers in public places, especially when using public networks for computer systems, we must pay attention to the security of personal information and computer systems, whether it is reading data or using computers to access the Internet, we must improve the safety in public places. Protection awareness; (3) Regularly perform virus detection or security detection on the computer system, so that problems in the computer system can be discovered in time or the virus can be found at an early stage, and then the virus can be detected and killed in time to solve the problem in time to prevent virus Step into a computer system, resulting in more damage and influence to effectively avoid the problem.

\section{Conclusion}

With the continuous improvement of China's economic level, the computer age is approaching, the development level of computers is getting higher and higher, and the upgrading is getting faster and faster. However, the problem that has been plagued by computers that have not been effectively solved is the maintenance and management of computer software systems. Problem. At present, computers have been widely used and applied in a wider range. Effective solution to the problems of computer software system maintenance and management can promote the optimization of computer performance and clear the obstacles for the use of computers.

\section{References}

[1] Huang Naifu. Research on daily management and maintenance of computer software [J]. Digital Technology and Application, 2014, 6: 223+225.

[2] Wang Yiguo. Research on the daily management and maintenance of computer software [J]. Network Security Technology and Application, 2013, 10:15+14.

[3] Liu Yue. Analysis of daily management and maintenance strategy of computer software [J]. Silicon Valley, 2015, 4: 112+111.

[4] Sheng Qi. Preliminary Study on Computer Software Maintenance Technology [J]. China E-Commerce, 2013 (08).

[5] Abdullah Makati. Research on Computer Software Maintenance and Management [J]. Information and Computer, 2015(17). 